\title{
Metabolismo e respiraçào do peixe-boi da Amazônia (Trichechus inunguis) ${ }^{(1)}$
}

\author{
G. J. Gallivan $\left(^{2}\right]$ \\ R. C. Best $\left({ }^{3}\right)$
}

\section{Resumo}

Estudo da respiração e metabolismo de peixesbois da Amazônia (Trichechus inunguis) pesando entre 20 e $170 \mathrm{~kg}$. Esses animais tễm uma taxa metabólica baixa, 0,36 vezes daquele prognosticado pela equação de Kleiber (1975) para mamíferos placentários. Eles têm também uma frequêencia respiratória e ventilaçảo minuto muito baixas e extração de $\mathrm{O}_{2}$ e produção de $\mathrm{CO}_{2}$ altos. O tempo médio de mergulho foi menos de 2 minutos, mas mergulhos de mais de 10 minutos de duração foram notados. Tempos de mergulhos seqũenciais mostraram um certo padrăo, somente no fato de que um mergulho prolongado é seguido por mergulhos de curta duraçăo.

\section{INTRODUÇ̧̃̃o}

Scholander \& Irving (1941) conduziram o único estudo amplo de metabolismo e respiração em Sirênios. Eles verificaram que o peixeboi da Flórida (Trichechus manatus) apresentava uma taxa metabólica e ventilaçăo minuto baixas e uma extraçăo de $\mathrm{O}_{2}$ alta. Como eles também encontraram volumes sangüíneos e concentraçōes de hemoglobina e mioglobina baixos, em relação a outros mamíferos marinhos. a taxa metabólica baixa foi considerada como sendo o fator que permitia aos peixesbois mergulhos de mais de 16 minutos de duração.

As baixas concentraçōes de hemoglobina e mioglobina, medidos por Scholander \& Irving, têm sido encontradas por outros pesquisadores (Blessing, 1972; White et al., 1976; Farmer et al.. 1979), mas não há nenhuma informação de medidas de outros parâmetros, desde o trabalhe original de Scholander e Irving (1941). O presente estudo é um reexame de suas medidas de metabolismo e ventilaçăo. Contudo, em contraste com o estudo de Scholander \& Irving (1941), que usaram animais presos e amarrados, os animais de nosso estudo podiam mergulhar livremente como eles preferissem. Tal situação fornecia valores próximos aos encontrados sob condições naturais.

\section{MATERIAL E MÉTODOS}

Este estudo foi conduzido, utilizando-se 6 peixes-bois da Amazônia (Trichechus inunguis) que foram capturados quando "bezerros" e mantidos em cativeiro. As características físicas dos animais são mostradas na Tabela 1.

As medidas foram tomadas com os animais em um tanque de $2,75 \times 1,75 \mathrm{~m}$ com água de uma profundidade de $1,0 \mathrm{~m}$. Para restringir a natação livre dos 5 animais menores, uma divisão reduziu ainda mais as dimensões do tanque para $2,3 \times 0,8 \mathrm{~m}$. A temperatura da água no tanque variava entre $26^{\circ}$ e $29^{\circ} \mathrm{C}$ que eram similares às temperaturas nos grandes tanques externos onde normalmente os animais eram mantidos.

Durante as medidas, o tanque era coberto por uma chapa de madeira compensada, submersa a $5 \mathrm{~cm}$. Uma parte da madeira compensada foi substituída por uma placa de acrílicc contendo uma máscara respiratória, como mostra a Fig. 1. No experimento inicial com os animais de $n$ ? 11, 13, 16 e 19, válvulas " $V$ " (Warren E. Collins, Braintree, Massachusetts) foram adaptadas à máscara. Contudo, estas eram resistentes aos grandes fluxos de ar e no experimento posterior foram substituídas por válvuia tipo Lloyd, construída em nossa oficina. A válvula tipo Lloyd apresentava uma resistência menor que $1 \mathrm{~cm} \mathrm{H} \mathrm{H}_{2} \mathrm{O}$, enquanto os

(1) - Versẩo original inglesa publicada em Physiolog'cal Zoology 53 (3): 245-253. University of Chicago Press.

(2) - Department of Anaesthesia, McMaster Univers ty, Hamilton, Ontario, Canadá.

(3) - Instituto Nacional de Pesquisas da Amazônia, Manaus. 
TABELA 1 - Características físicas dos peixes-bois utilizados neste estudo.

\begin{tabular}{|c|c|c|c|c|c|}
\hline $\begin{array}{l}\text { ANIMAL } \\
\text { (N.) }\end{array}$ & SEXO & $\begin{array}{l}\text { NÚMERO DE } \\
\text { COLETAS }\end{array}$ & $\begin{array}{l}\text { PESO } \\
\text { (KG) }\end{array}$ & $\begin{array}{l}\text { COMPRIMENTO* } \\
\text { (CM) }\end{array}$ & $\begin{array}{l}\text { IDADE } \\
\text { (ANO) }\end{array}$ \\
\hline \multirow[t]{2}{*}{01} & $M$ & 19 & 80 & 162 & 5 \\
\hline & & 7 & 87 & & \\
\hline \multirow[t]{2}{*}{11} & $M$ & 17 & 35 & 129 & 1 \\
\hline & & 7 & 36 & & \\
\hline \multirow[t]{2}{*}{13} & $M$ & 20 & 88 & 177 & 2 \\
\hline & & 8 & 105 & & \\
\hline \multirow[t]{2}{*}{16} & $M$ & 13 & 51 & 143 & 1 \\
\hline & & 7 & 65 & & \\
\hline \multirow[t]{2}{*}{18} & $M$ & 20 & 164 & 220 & ca 8 \\
\hline & & 8 & 177 & & . \\
\hline 19 & $M$ & 12 & 20 & 113 & 1 \\
\hline
\end{tabular}

(*) - o comprimento foi medido ao longo da curvatura dorsal, da ponta do nariz à ponta da cauda.

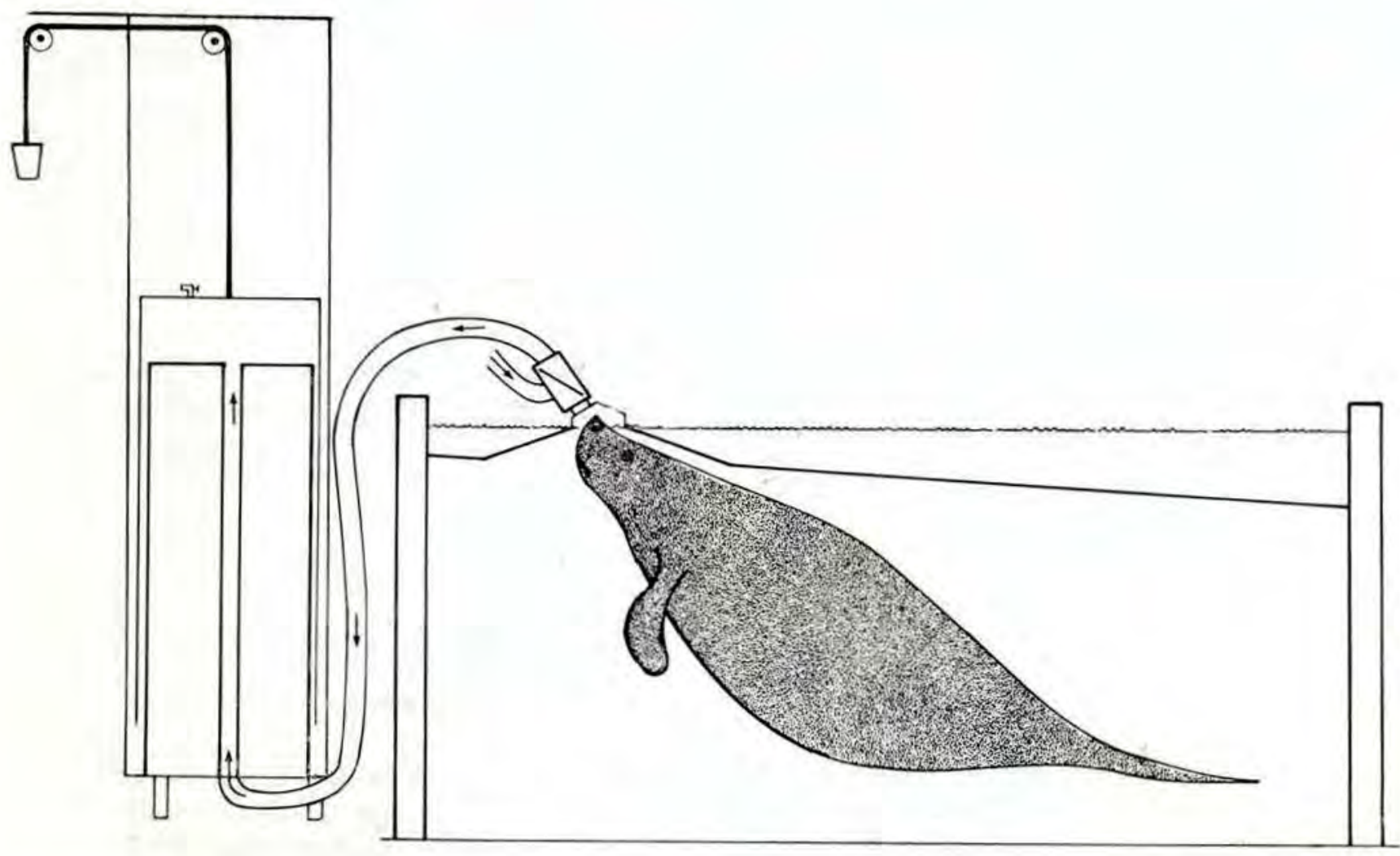

Fig. 1 - Desenho do sistema de tanques e espirômetro utilizados para se tomarem as medidas metabólicas e respiratórias. 
animais estavam respirando. O espaço morto máximo da máscara da válvula tipo Lloyd era de $250 \mathrm{ml}$.

O lado expiratório das válvulas era conectado por encanamento PVC ao espirômetro, para a coleta das amostras do gás expirado. $O$ conteúdo de $\mathrm{O}_{2}$ e $\mathrm{CO}_{2}$ dessas amostras foi determinado utilizando-se um analisador Scholander 0,5 cc (Scholander, 1947).

\section{PROCEDIMENTO EXPERIMENTAL}

Um registro diário constante foi usado durante 0 experimento. Aproximadamente às 21:00 $\mathrm{h}$ da noite precedente, a profundidade da água era ajustada a $0,8 \mathrm{~m}$ e a placa de madeira compensada era abaixada, a uma posição que deixava um espaço aéreo de $0,15 \mathrm{~m}$ entre a placa e a água. Às 08:00 horas da manhã do experimento, o tanque era cheio até o nível da máscara. O animal era, então, deixado por aproximadamente $2 \mathrm{~h}$ para se ajustar à situa ção, antes de se iniciarem as coletas de gás. As coletas de gás foram feitas por períodos de $30 \mathrm{~min}$., com um mínimo de $30 \mathrm{~min}$. entre as coletas. Normalmente, havia 4 períodos de coletas por dia, mas o número variava entre 2 a 5 . Durante os períodos de coleta, um observador anotava a duração entre as respiraçōes e o volume de cada expiração.

C experimento era coletado às $15: 00 \mathrm{~h}$ de cada dia. Depois disso, o tanque era limpo e o animal alimentado. Para minimizar o efeito da ação dinâmica específica, qualquer resto de comida era removido antes de a placa ser baixada, em preparação para os experimentos dos dias seguintes.

Com exceção do $n^{\circ} 19$, todos os animais foram testados em duas ocasiões separadas (Tabela 1). O teste inicial com o animal $n$ \% 11 foi conduzido para definir o tempo requerido por um indivíduo para aclimar-se à situação do experimento. As coletas de gases começaram no primeiro dia em que o animal foi introduzido à situação de experimento e continuado por 8 dias consecutivos. Baseado nos resultados obtidos com esse animal, um mínimo de 3 dias foi dado para a aclimação, antes das coletas de gases serem feitas com qualquer dos outros animais.

\section{Resultados}

Os resultados das medidas das séries iniciais com o animai $n^{0} 11$ săo mostrados na Figura 2. Teste de aleatorização seqüencial, usando o teste de diferença sucessiva pelo processo dos quadrados mínimos (Zar, 1974), mostraram volume corrente $\left(V_{T}\right)$ e proporção de troca respiratória $(R)$ foram aleatórios no tempo, mas consumo de oxigênio $\left(\mathrm{V}_{2}\right)$, freqüência respiratória $\left(f_{R}\right)$ e ventilação $\left(V_{E}\right)$ não o foram. O teste de Duncan (Zar, 1974) mosirou que valores para $\mathrm{V}_{2}$, nos dois primeiros dias, diferiram significativamente dos valores dos dias subseqüentes. Os valores para $f_{R}$ e $V_{E}$ nos três primeiros dias foram significativamente diferentes daqueles dos dias
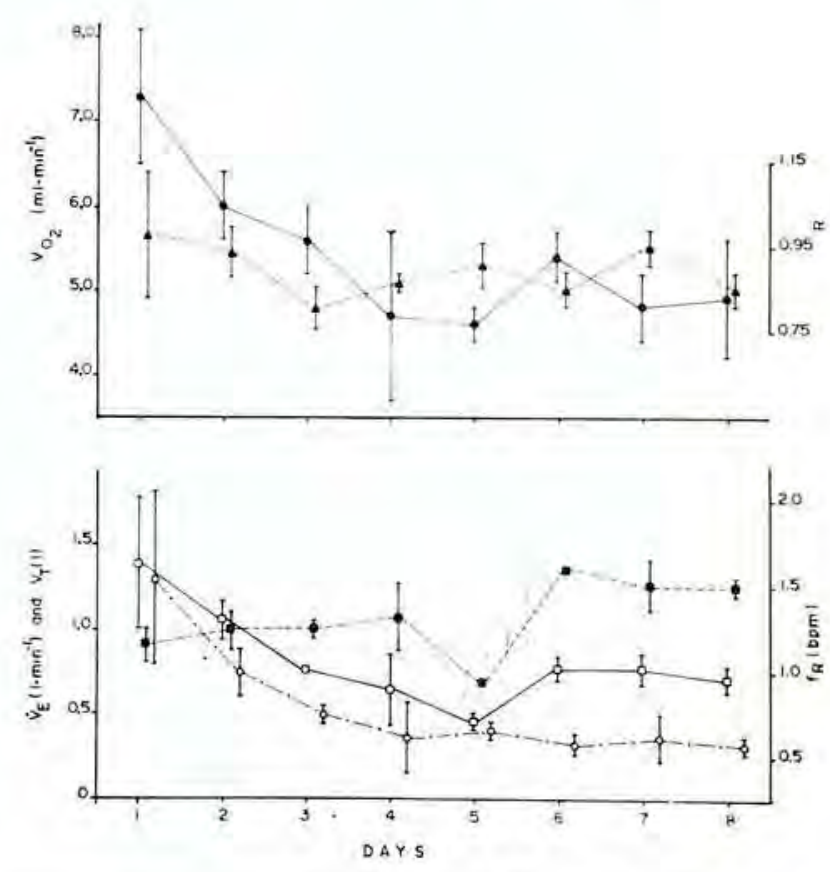

Fig. 2 - Medidas metabólicas e respiratórias obtidas com o animal $n .^{\circ} 11$, durante a primeira série de medidas.

$$
\begin{aligned}
& \text { No gráfico superior } \mathrm{O}=\mathrm{V}_{\mathrm{O}_{2}} \\
& \begin{aligned}
\boldsymbol{\nabla} & =\mathrm{R} \\
\text { No gráfico inferior } \boldsymbol{\nabla} & =\mathrm{V}_{\mathrm{T}}
\end{aligned} \\
& \mathrm{O}=\mathrm{f}_{\mathrm{R}} \\
& \square=\mathrm{V}_{\mathrm{E}}
\end{aligned}
$$

As variaçōes no $\mathrm{V}_{\mathrm{O}_{2}}, \mathrm{~V}_{\mathrm{E}}$ e $\mathrm{f}_{\mathrm{R}}$ com o tempo são significativos. Os valores são apresentados com média \pm desvio padrão. 
subseqüentes. Assim, para análises ulteriores desta série de medidas, somente valores dos últimos cinco dias foram utilizados.

A significância das mudanças no tipo de válvulas e no peso ganho pelos indivíduos, entre a primeira e segunda série de medidas, foi testada usando-se o test-t. Dentro do grupo, nenhuma diferença significativa foi notada, conseqüentemente os resultados da primeira $e$ segunda série de medidas para cada animal foram agrupados. Os pesos foram recalculados como peso médio da primeira e sequnda série.

A relação entre a taxa metabólica, expressa em Kcal/dia $\left(\mathrm{Kcal} /\right.$ dia $\left.=\mathrm{VO}_{2}\right) \quad\left(1 \cdot \mathrm{min}^{-1}\right) \times$ $60 \mathrm{~min} . \times 24 \mathrm{~h} \times 4,825 \mathrm{Kcal} \cdot 1^{-1}$ ), e peso é mostrada na Fig. 3. A equação relacionando taxa metabólica (MR) e peso do corpo (W) foi $M R=30 W^{0,28}$. O exponente não foi significativamente diferente da equação de Kleiner (1975), MR $=10 \mathrm{~W}^{0,75}$, mas o traço foi significativamente inferior, sendo os valores medidos

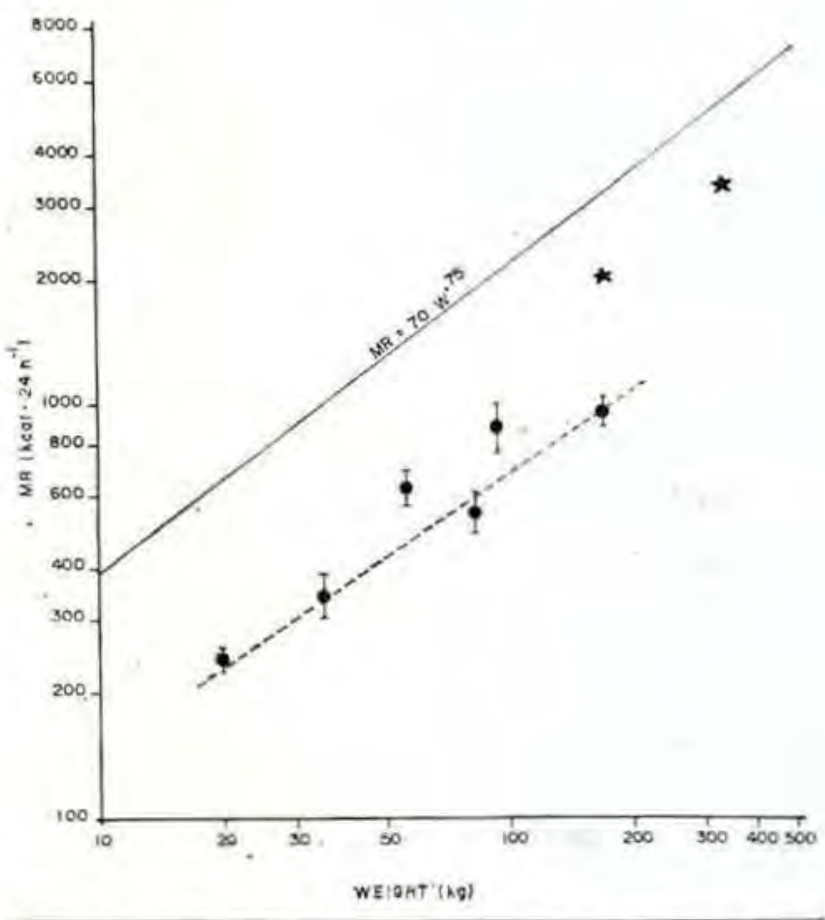

Fig. 3 - A taxa metabólica média (MR) dos animais deste estudo são relacionadas com o peso. Os valores são relacionados com a média \pm desvio padrão e a linha pontilhada é derivada da equaçāo $\mathrm{MR}=30 \mathrm{~W}^{0} 68$. A linha continua é a equação de Kleiber (1975) para a comparaçâo interespecífica das taxas metabólicas. As estrelas sẩo as taxas metabólicas medidas por Scholander \& Irving (1941). igual a $0,36 \pm 0,06(n=6)$ vezes daqueies prognosticados pela equação de Kleiber.

As relações entre as outras variáveis respiratórias $\left(V T, f_{R}, V_{E}, R\right)$, diferença entre $\mathrm{O}_{2}$ inspirado e expirado $\left(\mathrm{Fl}-\mathrm{EO}_{2}\right)$ e concentrações de $\left.\mathrm{CO}_{2}\left(\mathrm{FE}-\mathrm{I} \mathrm{CO}_{2}\right)\right)$ e peso são mostradcs na Fig. 4. A proporção de troca respiratória não foi correlacionada com peso e $f_{R}$ diminuiu com $\sigma$ peso. Todas as outras variáveis aumentaram com o peso e os coeficientes de regressão são dados na Tabela 2.

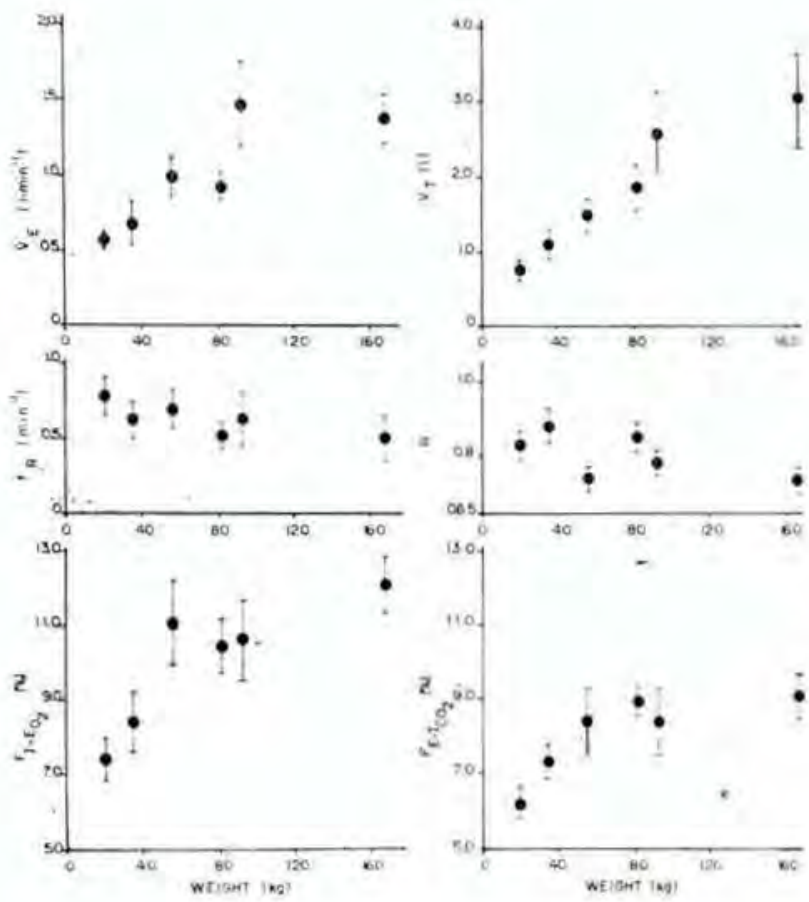

Fig. 4 - Valores para os vários parâmetros respiratórios são relacionados com o peso do animal. Os valores săo média \pm desvio padrāo.

A pressão de $\mathrm{O}_{2}$ alveolar no término de mergulho ( $\mathrm{PA}$ ) e pressão de $\mathrm{CO}_{2}(\mathrm{PA})$ foram calculados durante a segunda série de medidas para cada indivíduo. Os resultados são apresentados na Fig. 5. Os cálculos de PA e PA foram feitas supondo-se que a $\mathrm{O}_{2} \quad \mathrm{CO}_{2}$

difusão e a mistura cardiogênica produziriam uma mistura homogênea de gases dentro do pulmão e vias aéreas durante a retenção da respiração. Testes subseqüentes, nos quais 
TABELA 2 - Os coeficientes de regressão para as relações entre os vários parâmetros respiratórios e peso. As equaçōes estāo na forma $x=a W b$.

\begin{tabular}{|c|c|c|c|c|c|c|}
\hline PARĀMETRO & $\mathrm{N}$ & a & b & $r^{2}$ & $\mathrm{FI}_{4}$ & EPE \\
\hline$v_{T}$ & 6 & 0,094 & 0.68 & 0,97 & 121,36 & 0,045 \\
\hline$v_{E}$ & 6 & 0,14 & 0,46 & 0,85 & 22,42 & 0,072 \\
\hline$f_{R}$ & 6 & 1,35 & $-0,20$ & 0.74 & 11,28 & 0.043 \\
\hline $\mathrm{F}_{1-\mathrm{E}_{\mathrm{O}_{2}}}$ & 6 & 3,63 & 0,23 & 0,87 & 27,47 & 0,032 \\
\hline${ }^{\mathrm{F}-1} \mathrm{CO}_{2}$ & 6 & 3,58 & 0,18 & 0,87 & 25,84 & 0,026 \\
\hline
\end{tabular}

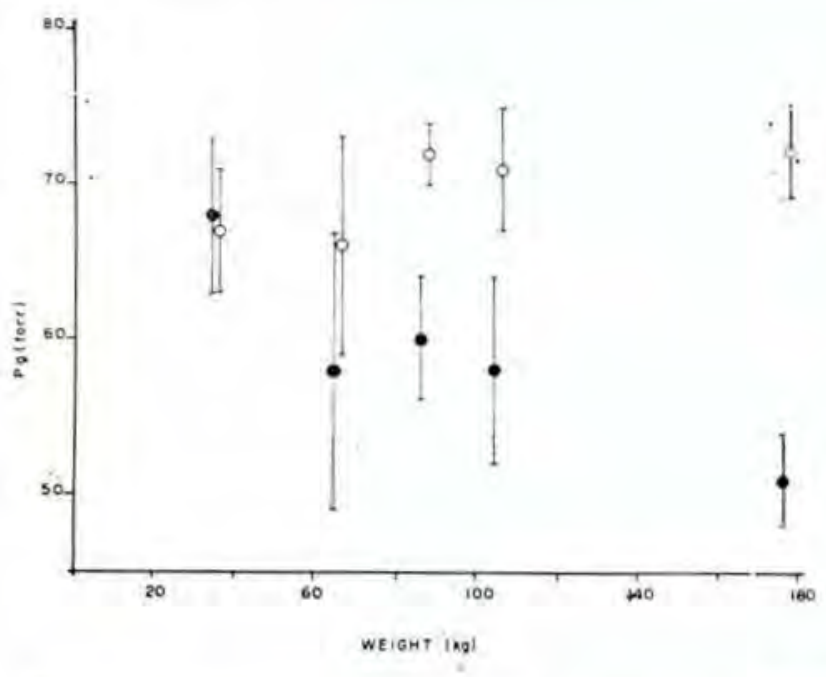

Fig. 5 - Pressão de $\mathrm{O}_{2}$ alveolar de término de mergutho $(0)$ e $\mathrm{CO}_{2}(0)$ sã̃o relacionados com o peso do animal. Os valores são média \pm desvio padrăo.

amostras de término respiratório, por várias respiraçōes, foram tomadas da máscara e comparadas à amostra expiratória misturada, coletada no expirômetro durante o mesmo período, confirmaram a suposiçăo acima (Gailivan, Kanwisher \& Best, não publicado). A equação de regressão para $\mathrm{PA}$ versus peso foi $\mathrm{PA}$ $\mathrm{O}_{2}$

$=69-0,1 \mathrm{~W}\left(\mathrm{r}^{2}=0,82 ; \mathrm{F}_{3}^{\prime}=14,89 ; \mathrm{EPE}=3\right)$

e para $\mathrm{PA}$ versus peso foi $\mathrm{PA}=65+$ $0,05 \mathrm{~W}\left(r^{2}=0,74 ; \mathrm{F}_{3}=8,49 ; \mathrm{EPE}=2\right)$.
Para cada indivícuo, as relações entre variáveis foram examinadas, usando-se o coeficiente de correlação $(r)$. A significância dessas correlações foram testadas pela comparaçẫo do valor medio $r$ para todos os animais a zero, usando-se um test-t. Enquanto diversas variáveis mostraram correlaçōes significativas para cada individuo, houve somente duas correlações de significância para todos os animais. $\mathrm{O}$ consumo de oxigênio foi correlacionado com $\left.\mathrm{V}_{\mathrm{E}} \overline{i r}=0.68\right)$, e $\mathrm{V}_{\mathrm{T}}$ foi negativamente correlacionado com $f_{R}(\bar{r}=0,74)$.

Os animais deste estudo respiravam somente uma vez a cada vez que vinham à superficie, permitindo uma fácil anotação do tempc de mergulho $\left(t_{\mathrm{d}}\right)$ e volume expirado. O tempo médio de mergulho foi de menos de 2 minutos mas os tempos de mergulho eram altamente variáveis, atingido 5 segundos a 10 minutos (Fig. 6) .

Para permitir um exame mais detalhado da variação seqüencial e padrōes de $t_{d}$ e VT, 10 períodos de coletas, para cada animal, foram escolhicias. Essas variáveis foram, então, testadas por aleatorização seqüencial, periodicidade, distribuiçăo e correiação. O teste de diferença sucessiva pelo processo dos quadrados mínimos revelou que ambas as variáveis eram seqüencialmente ao acaso, dentro do período

- EPE = erro padrăo do estimativo 


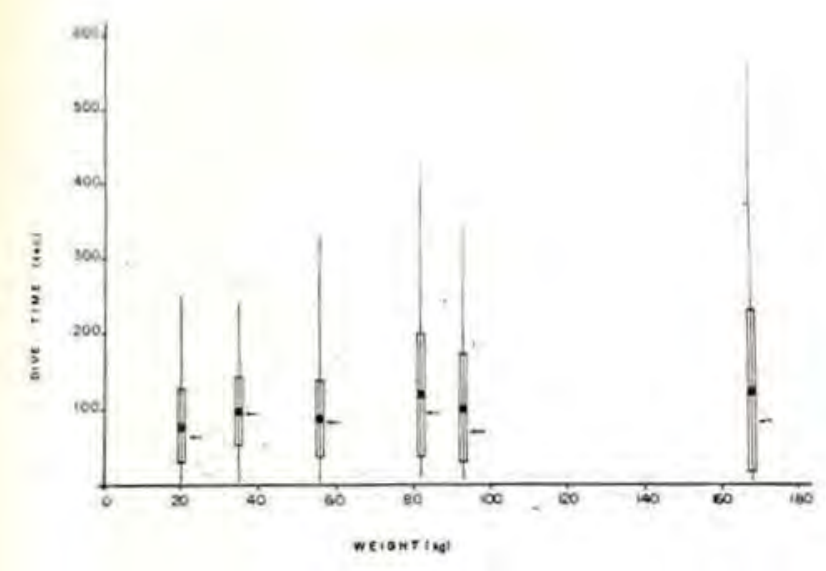

Fig. 6 - Diagrama do tempo de mergulho versus o peso do animal. As barras negras são o tempo de mergulho médio $e$ as barras brancas são 1 desvio padrão. As linhas indicam a gama $\lambda$ dos tempos de mergulhos observados e as setas apontam a mesma do tempo de mergulho.

de coleta. Um teste seriado aleatorizado para periodicidade (Ellis et al., 1978), mostrou que nenhuma das variáveis era cíclica. Uma comparação de $\bar{x}$ e $s^{2}(Z a r, 1974)$ revelou que $t_{d}$ tinha uma distribuiçăo aglomerada enquanto a distribuição de $V_{T}$ era uniforme. Nāo foi encontrada nenhuma correlação entre $t_{d}$ e VT.

Tempos de mergulho não eram distribuídos normalmente mas eram assimétricos com a mediana sendo menor que a média (Fig. 6) . Isso e a distribuição aglomerada, dentro dos períodos de coleta, indicaram que algum fator estava influenciando no tempo de mergulho. Uma possibilidade era de que o efeito restante de um mergulho prolongado poderia influenciar na duração dos mergulhos subseqüentes. Para examinar isso, os mergulhos mais longos, correspondentes a $5 \%$ dos mergulhos totais, para cada indivíduo, foram escolhidos A duração dos 6 mergulhos seguindo merguIhos prolongados foram, então, examinados. A duração dos seis mergulhos năo foram estatisticamente diferentes. O primeiro mergulho, seguindo um mergulho prolongado, foi normalmente mais curto do que os mergulhos subseqüentes, com $67 \%$ dos primeiros mergulhos. tendo menos de 1 minuto de duração. Contudo, a gama de valores era grande e mergulhos tão prolongados quanto os longos mergulhos anteriores foram notados.

\section{DISCUSSÃo}

O peixe-boi da Amazônia é caracterizado pela taxa metabólica baixa, ventilaçăo minuto e freqüência respiratória baixas, e uma tolerância para níveis baixos de $\mathrm{O}_{2} \mathrm{e}$ nível alto de $\mathrm{CO}_{2}$. Apesar de possuirem capacidade para realizarem mergulhos de acima de 10 minutos de duração, eles normalmente mergulham por menos de 2 minutos.

Os tempos de mergulho neste estudo eram similares àqueles apresentados por esses animais nos tanques de criaçẩo maiores e para tempos de mergulho observados em outros sirênios sob ambas as condições: natural e em cativeiro (Parker, 1922; Scholander \& Irving. 1941; Kenny, 1967; Hartman, 1971; Anderson \& Birtles, 1978). Kenny (1967) e Hartman (1971) sugeriram que sirênios eram mergulhadores cíclicos. Os resultados de nosso estudo não apoiam essa suposição e uma reanálise dos resultados de Hartman (1971) também faIhou em fornecer evidência de ciclos. Em contraste, os padrões de mergulho dos peixesbois são seqüencialmente ao acaso, mas mergulhcs prolongados, que têm uma maior depleção de $\mathrm{O}_{2}$ e acúmulo de produtos metabólicos finais, são geralmente seguidos por uma série de mergulhos curtos, durante os quais qualquer débito de $\mathrm{O}_{2}$ é compensado (Kanwisher et al., 1980). Isso produz uma distribuiçăo aglomerada de tempos de mergulho, quando os merguIhos curtos excedem grandemente os merguthos prolongados.

Uma comparação do comportamento de mergulho dos peixes-bois, com aquele de outros mamíferos marinhos, é difícil, pois a maioria da literatura sobre mergulho tem se concentrado nos tempos de mergulho máximo ou tratam somente de mergulhos selecionados. A literatura sobre sirênios é única e a maior parte da informação sobre função respiratória é sobre freqüência respiratória e tempos de mergulho de animais livres (Dextler \& Freund, 1906: Parker, 1922; Scholander \& Irving, 1941: Kenny, 1967; Hartman, 1971; Anderson \& Birtles, 1978). Contudo, algumas informações comparảveis estão disponíveis nos estudos de Pasche (1976), Parsons (1977) e Gallivan (1977) sobre focas. Os peixes-bois tendem a 
fazer mergulhos mais curtos, mas essa diferença provavelmente reflete mais numa diferenca no padrão respiratório do que na capacidade de mergulho. As focas respiram várias vezes depois de um mergulho, enquanto que um peixeboi respira somente uma vez e os reajustes metabólicos seguintes ao mergulho são feitos durante uma série de mergulhos curtos, em vez de durante um período longo de respiração. Isso diminuirá grandemente o tempo médio de mergulho dos peixes-bois.

Os cetáceos, que também mergulham com inspiração (Kooyman, 1973), têm um padrão respiratório muito similar ao dos peixes-bois. O volume corrente dos peixes-bois, neste estudo, foi similar ao dos Delfinidae, na mesma faixa de peso (Karandeeva et al., 1973). A taxa metabólica, ventilação minuto e freqüência respitatória dos peixes-bois são significativamente mais baixas. É provável que a taxa metabólica mais baixa dos peixes-bois é o fator que lhes dá uma capacidade relativamente maior de mergulho. Enquanto os golfinhos têm uma depleção virtual de $\mathrm{O}_{2}$ dentro dos 4 minutos de submersão (Ridgway et al., 1969), os peixes-bois ainda têm $4 \%$ da concentração de $\mathrm{O}$. no pulmăo depois de 10 minutos de submersão (Kanwisher et al., 1980) .

O consumo fracional de $\mathrm{O}_{2}$ e a produçăo de $\mathrm{CO}_{2}$, neste estudo, foram mais altos do que os observados para peixes-bois e outros mamiferos marinhos mantidos à superfície da água (Scholander \& Irving, 1941). Os valores de $\mathrm{PA}$ P $\mathrm{PA}$ indicam que os peixes-bois $\mathrm{O}_{2} \quad \mathrm{CO}_{2}$

são tolerantes à severa hipoxia hipercápnica. Valores similares de PA têm sido observa. $\mathrm{O}_{2}$

dos depois de mergulhos em focas mas os valores de PA de focas são muito menores $\mathrm{O}_{2}$

(Gallivan, 1977). A similaridade de valores de PA é um resultado da forma sigmoidal da $\mathrm{O}_{2}$

curva de dissociação da oxihemoglobina, enquanto que os valores mais altos de PA

$\mathrm{CO}$, para peixes-bois, resultam da linearidade relativa da curva de dissociação de $\mathrm{CO}_{2}$ e das proporções mais baixas de $\mathrm{VE} / \mathrm{V}_{\mathrm{CO}_{2}}$ de peixes. bois, comparadas às focas.
As taxas metabólicas medidas, neste estudo, são metade daquelas medidas por Scholander \& Irving (1941). A diferença possivelmente é interespecífica, contudo, diferenças no procedimento experimental não podem ser ignoradas. No presente estudo, cuidados foram tomados para assegurar que os animais eram pós-absortivos e estavam aclimados à situação experimental e às condições térmicas. Em contraste, os animais usados por Scholander \& Irving (1941) eram recém-capturados (menos de duas semanas) e eram presos a uma prancha para o estudo. Eles observaram uma freqüência respiratória aumentada relacionada às observaçôes feitas quando os animais eram livres. As taxas metabólicas eram similares às medidas com o animal no 11 , no seu primeiro dia de situaçäo experimental (Fig. 2). Irving (1970) justificou o sisiema de restrição física, usado por Scholander \& Irving (1941), dizendo que animais restritos eram metabolicamente imperturbados porque eles aceitavam a máscara respiratória. Contudo, o animal no 11 permaneceu calmamente durante todo o experimento, mas sua taxa metabólica, ventilação minuto e freqüência respiratória declinaram significativamente durante os três primeiros dias. Um processo similar tem sido observado em focas (Gallivan, não publicaco) e inćica que um período de aclimação é necessário para superar a tensão associada ao experimento, mesmo com animais sujeitos a uma restrição mínima.

Não obstante a variação na idade e tamanho dos peixes-bois utilizados neste estudo, o exponente de peso específico, para taxa metabóíca, não é significativamente diferente daquele da equação de Kleiber (1975). Como animais mais jovens normalmente têm uma taxa metabólica mais alta (Kleiber, 1975), esperava-se um exponente mais baixo. Contudo. Brody (1964) e Thonney et al. (1976) fornecem informação de que o exponente é especifico para espécie e há considerável variabilidade entré espécie. O exponente deste estudo pode ser especifico para peixes-bois ou pode refletir o fato de que todos os animais eram machos, consumindo uma dieta adulta. 


\section{AgRAdeCIMENTOS}

Os autores desejam agradecer ao Dr. W. E. Kerr, Dr. W. J. Junk, Sr. Pedro Makiyama e ao pessoal do Projeto Peixe-Boi e Divisão de Peixe e Pesca do INPA, por seu apoio, também a Megumi Yamakoshi, pela tradução e datilografia co manuscrito e Dr. F. Pimentel de Souza pelas críticas no manuscrito. Esta pesquisa foi financiada pelo CNPq (Brasil), Vancouver Públic Aquarium (Canadá), Fauna Preservation Seciety (UK), World Wildlife Fund (U.S) e IBDF (Brasil) .

\section{SUMMARY}

A study was made of respiration and metabolism in Amazonian manatees (Trichechus inunguis) weighing between 20 and $170 \mathrm{~kg}$. Thse animals had a low metabolic rate, 0.36 times that predicted by Kleiber's (1975) equation for placental mammals. They also had a low breathing frequency and minute ventilation, and high $\mathrm{O}_{2}$ extraction and $\mathrm{CO}_{2}$ output. The mean dive times were less than 2 minutes but dives of up to $10 \mathrm{~min}$. duration were noted. Sequential dive times exhibited a pattern only in that several short dives followed a prolonged dive.

\section{REFERÊNCIAS BIBLIOGRÁFICAS}

ANDERSON, P.K. \& BIRTLES, A.

1978 - Behaviour and ecology of the dugong, Dugong dugon (Sirenia): observations in Shoalwter and Cleveland Bays, Queensiand. Aust. Wildl. Res., 5: 1-23,

BLESSING, M.H.

1972 - Studies on the concentration of myoglobin in the sea-cow and porpoise. Comp. Biochem. Physiol, , 41A: 475-480.

BRODY, S.

1964 - Bioenergetics and growth. New York. Hafner Publ. Co. 1023 D.

Dextler, H. \& Freund, L.

1906 - Contributions to the physiology and biology of the dugong. Am. Nat., 40: 49-72.

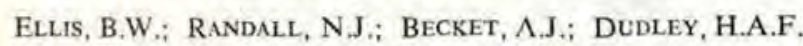
1978 - Continuous blood sampling and time series analysis in endocrine research. J. Med. Eng. Eech., 2: 195-199.

FARMER, M.; WEBER, R.E.; BONAVENTUKA, J.; BEST, R.C.; DOMNING, D.

1979 - Functional properties of the hemoglobin and whole blood in an aquatic mammal, the Amazonian manatee (Trichechus inunguis). Comp. Biochem. Physiol,, 62A: 231-238.
GaLlivan, G.J.

1977 - Temperature regulation and respiration in freely diving harp seals (Pho:a groenlandica). M. Sc. Thesis, Univ, of Guelph, Guelph, Ontario, Canada, $60 \mathrm{p}$.

HARTMAN, DS.

1971 - Behavior and ecology of the Florida ma. natee, Trichechus manatus latirostris (Harlan), at Crystal River, Citrus County. Ph. D. Thesis. Cornell University, Ithaca, New York, $285 \mathrm{p}$.

IRVING, L.

1970 - Morpho-physiological adaptations in marine mammals for life in polar areas. In: Holdgate, M.W. ed. Antarctic ecology. New York. Academic Press, p. 455-463.

KANwisher, J.; Best, R.C.; Gallivan, J.

1979 - The aquatic physiology of the Amazonian manatee (Trichechus inunguis). in prep.

Karandeeva, O.G.; Matisheya, S.K.; Shapunov, V.M. 1973 - Features of external respiration in the delphinidae. In: Chapskii, K.K. \& Sokolov. V.E. eds. Morphology and ecology of marine mammals. New York. J. Wiley and Sons, p. 196-206.

KENNY, R.

1967 - The breathing pattern of the dugong. Aust. J. Sci., 29: 372-373.

KLEIBER, M.

1975 - The fire of life, 2 ed. New York. Kreiger Publ. Co., 453 p.

KoOYMAN, G.L.

1973 - Respiratory adaptations of marine mammals. Am. Zool., 13: 457-468

PARKER, G.H.

1922 - The breathing of the Florida manatee (Trichechus latirostris). J. Mamma!., 3: 127135.

PARSONS, J.

1977 - Metabolic studies on ringed seals (Phoca hispida). M. Sc. Thesis. Univ. of Guelph, Guelph, Ontario, 82 p.

PASCHE, A.

1976 - The effect of hypercapnia on respiratory characteristics and diving behaviour of freely diving seals. Respir. Physiol,, 26: 183-194.

RIDGWAY, S.H.; SCRONCE, B.L.; KANWISHER, J.

1969 - Respiration and deep diving in the bottlenose porpoise. Science 166: 1651-1654.

SCHOL_ANDER, P.F.

1947 - Analyzer for accurate estimation of respiratory gases in one-half cubic centimeter samples. J. Biol. Chem., 167: 235-250. 
SCHOLANDER, P.F. \& IRVING, L.

1941 - Experimental investigations on the respiration and diving of the Florida manatee. J. Cell. Comp. Physiol., 17: 169-191.

THONNEY, M.L.; TOUCHBERRY, R.W : GOODRICH, R.D.; MEISKE, J.C.

1976 - Intraspecies relationship between fasting heat production and body weight: a reevaluation of W0.57. J. Anim. Sci., 43: 692. 704.
WhItE, J.R.; HARKNESS, D.R.; ISAAKS, R.E.; DUFFIELD, D.A.

1976 - Some studies on blood of the Florida ma. natee, Trichechus manatus latirostris. Comp. Biochem. Physiol., 55A: 413-417.

ZAR, J.H.

1974 - Biostatistical analysis. England Cliffs, New Jersey. Prentice-Hall, $620 \mathrm{p}$.

(Aceito para publicação em $07 / 12 / 80$ ) 